\title{
FossiL: A Robust Relational Learner
}

\author{
Johannes Fürnkranz \\ juffioai.univie.ac.at \\ Austrian Research Institute for Artificial Intelligence \\ Schottengasse 3 \\ A-1010 Vienna \\ Austria
}

\begin{abstract}
The research reported in this paper describes FossiL, an ILP system that uses a search heuristic based on statistical correlation. This algorithm implements a new method for learning useful concepts in the presence of noise. In contrast to Fon's stopping criterion, which allows theories to grow in complexity as the size of the training sets increases, we propose a new stopping criterion that is independent of the number of training examples. Instead, Fossn's stopping criterion depends on a search heuristic that estimates the utility of literals on a uniform scale. In addition we outline how this feature can be used for top-down pruning and present some preliminary results.
\end{abstract}

\section{Introduction}

Being able to deal with noisy domains is a must for learning algorithms that are meant to learn concepts from real-world data. Significant effort has been made into investigating the effect of noisy data on attribute-value learning algorithms (see e.g. [22, 3, 4, 17]). Not surprisingly, noise handling methods have also entered the rapidly growing field of Inductive Logic Programming [15]. Linus [16] relies directly on the noise handling abilities of decision tree learning algorithms, others, like $m$ Foll [9] and REP [5], have adapted well-known methods from attribute-value learning for the ILP framework.

This paper presents FossiL, a FoIL-like algorithm [20] that uses a search heuristic based on statistical correlation (Sect. 2). One of the nice features of this heuristic is that it gives a reliable measure of the heuristic value of a literal on an absolute and uniform scale. We show empirically that this feature can advantageously be used to deal with noise by cutting off all literals that have a heuristic value below a certain threshold (Sect. 3). We also present empirical evidence that this threshold is robust, in the sense that a good value for it is independent of the number of training examples and of the amount of noise in the data (Sect. 4). After comparing Fossil to FoIL and $m$ FoIL we introduce several ideas for adapting pruning methods from decision tree learning in a topdown fashion along with some preliminary results (Sects. 5 and 6 ) and finally draw some conclusions (Sect. 7). 


\section{FossiL's Search Heuristic}

\subsection{The Correlation Heuristic}

FossiL's evaluation function is based on the concept of statistical correlation. The correlation coefficient of two random variables $X$ and $Y$ is defined as

$$
\operatorname{corr}(X, Y)=\frac{E\left(\left(X-\mu_{X}\right)\left(Y-\mu_{Y}\right)\right)}{\sigma_{X} \times \sigma_{Y}}=\frac{E(X \times Y)-\mu_{X} \times \mu_{Y}}{\sigma_{X} \times \sigma_{Y}}
$$

where $\mu$ and $\sigma$ are expected value and standard deviation, respectively, of the random variables $X$ and $Y$.

This correlation coefficient measures the degree of dependence of two series of points on a scale from -1 (negative correlation) to +1 (positive correlation). In the following description of its adaptation as a search heuristic for the Inductive Logic Programming algorithm FolL, we will follow the notational conventions used in [14].

Suppose FossiL has learned a partial clause $c$. Let the set of tuples $T_{c}$ of size $n(c)$, containing $n^{\oplus}(c)$ positive and $n^{\ominus}(c)$ negative instances, be the current training set. We arbitrarily assign the numeric values +1 and -1 for the logical values true and false. The variable $X$ in (1) now represents the truth values $V(c)$ of the tuples in $T_{c}$. The variable $Y$ denotes the the truth values $V(L)$ of a candidate literal $L$. A literal $L$ is said to be true, whenever there exists a tuple in $T_{c}$ that satisfies $L$; if $L$ introduces new variables, they must have at least one instantiation that makes the literal true. Note that $V(c)$ and $V(L)$ naturally contain the same number of values.

The expected values in (1) will be estimated by the mean values of $V(c)$ and $V(L)$ respectively. Standard deviation will be approximated by the empirical variance. Thus we get

$$
\begin{array}{cc}
\mu_{c}=\frac{n^{\oplus}(c)-n^{\ominus}(c)}{n}, \quad \sigma_{c}^{2}=E\left(V(c)^{2}\right)-E(V(c))^{2}=1-\mu_{c}^{2}, \\
\mu_{L}=\frac{n^{\oplus}(L)-n^{\ominus}(L)}{n}, \quad \begin{array}{c}
\sigma_{L}^{2}=1-\mu_{L}^{2} \\
\text { with } n=n(L)=n(c)=n^{\oplus}(c)+n^{\ominus}(c),
\end{array}
\end{array}
$$

The last remaining term to be computed is $E(V(c) \times V(L))$. If both the truth values $v(c)$ and $v(L)$ of a tuple and the literal under scrutiny have the same sign, then $v(c) \times v(L)=1$. Conversely, if one is positive and the other negative we have $v(c) \times v(L)=-1$. If we denote the number of positive tuples yielding a negative value for the literal $L$ with $n^{\oplus}(c)^{\ominus}$ (and analogously define $n^{\oplus}(c)^{\oplus}$, $n^{\ominus}(c)^{\oplus}$ and $\left.n^{\ominus}(c)^{\ominus}\right)$, we get

$$
E(V(c) \times V(L))=\frac{n^{\oplus}(c)^{\oplus}+n^{\ominus}(c)^{\ominus}-n^{\ominus}(c)^{\oplus}-n^{\oplus}(c)^{\ominus}}{n}
$$

The partial results of above now only need to be substituted into the formula for the correlation coefficient (1). As $\mu_{c}$ and $\sigma_{c}$ only need to be evaluated once 
for each tuple set $T_{c}$, evaluation of this formula is not as complicated as it may seem at first sight. Also notice that with this approach no separate calculation for negated literals has to be performed, as a high negative correlation indicates a high dependence on the negated literal.

The literal $L_{c}$ with the highest absolute value of the correlation coefficient (or $\neg L_{c}$ if the sign of the coefficient is negative) is then chosen to extend $c$ to form a new clause $c^{\prime}$. This is based on the assumption that its high correlation with the current training set $T_{c}$ indicates some form of causal relationship between the target concept and $L_{c}$. The set $T_{c}$ is then extended to a new set of tuples $T_{c^{\prime}}$ (which in general will have a different size) and the process continues as described in [20].

\subsection{Interesting Features of the Correlation Heuristic}

The information gain heuristic used in C4.5 [22] and FolL has been extensively compared to other search heuristics in decision tree generation $[18,6]$ and Inductive Logic Programming [14]. The general consensus seems to be that it is hard to improve on this heuristic in terms of predictive accuracy in learning from noise-free data. While our results confirm this, we nevertheless claim that FosSIL's evaluation function has some important features that distinguish it from the weighted information gain heuristic used in Foll.

- In Foll, the heuristic value of each literal and of its negation have to be calculated separately. FossiL does this in one calculation, as positive correlation indicates a causal relationship between the tuple set and the literal under scrutiny, while negative correlation indicates a causal relationship between the tuple set and the negation of the literal.

- The correlation function is symmetric and gives equal consideration to covering many positive and excluding many negative examples.

- The correlation between a tuple set and a literal that has at least one true grounding for each tuple is undefined, because $\mu_{L}$ will be 1 and thus $\sigma_{L}$ will be 0 (see (2)). Most of these literals are irrelevant, as they do not exclude any negative instances. However, some of them might be very useful, if they introduce new variables. The current version of FossiL ignores this problem by treating undefined cases as having correlation 0 and thus has severe problems in learning programs that need determinate literals [21]. Setting the heuristic value for the undefined cases to a value $D>0$ might be a simple solution for some of the pathological cases, because in that case FossIL would add literals with undefined correlation values whenever no other literal has a correlation $>D$. Irrelevant literals could be removed later in a post-processing phase. ${ }^{1}$

1 Not all of the problematic cases can be taken care of in this way: Quinlan's determinate literals are literals that have exactly one extension of each positive tuple and no more than one extension of each negative tuple. The undefined cases for the correlation value on the other hand are literals that have at least one extension for both positive and negative tuples. 
- FossiL's correlation coefficient - after taking absolute values and choosing the appropriate, positive or negative, literal - allows to compare the candidate literals on a uniform scale from 0 to 1 .

How this last property of the correlation heuristic can be used for a simple, but powerful criterion to distinguish noise from useful information will be described in Sect. 3.

\section{The Cutoff Stopping Criterion}

The value of FoIL's evaluation function is dependent on the size of the tuple set. The same literal will have different information gain values in different example set sizes of the same concept, although its relative merit compared to its competitors will be about the same. FossiL on the other hand can judge the relevance of a literal on an absolute basis. This allows the user to require the literals that are considered for clause construction to have a certain minimum correlation value - the cutoff.

This can be used as a simple, but robust criterion for filtering out noise, as it can be expected that tuples originating from noise in the data will only have a low correlation with predicates in the background knowledge. If no literal with a correlation above the cutoff can be added to the current clause, this clause is considered to be complete. Similarily, if no literal can be found that can start a new clause, the concept definition is considered to be complete. Note that it may happen that FossiL "refuses" to learn anything in cases where no predicate in the background knowledge has a significant correlation with the training data. ${ }^{2}$

If a clause that cannot be further extended still covers negative examples, FossiL follows a simple strategy: If the clause covers more positive than negative examples, it is retained, and the examples that are covered will be removed from the tuple set. If the clause covers more negative than positive examples, it will not be added to the concept description, and only the positive examples that would have been covered by this clause will be removed. This is in contrast to FolL, where learning stops entirely as soon as a clause is found that covers less than $80 \%$ positive examples. In that case Forl leaves the remaining positive examples uncovered, while FossiL further tries to find clauses that cover some of them. The fact that the learned clauses always have to cover more positive than negative examples guarantees that the algorithm used in FossiL can never produce a bigger error on the training set than the method used in FolL. It was mainly this improvement that lead to a relatively good performance of FossiL at tests on the mesh data ( $32 \%$ as opposed to $21 \%$ for $m$ Foll [9])

2 This has actually happened several times, and is evident in the result with $50 \%$ Noise (i.e. random classification) in Table 1 , where Fossil did not learn a single clause in any of the 10 training sets. 


\section{Experimental Evaluation}

\subsection{Setup of the Experiments}

For the experiments in this paper we have used the domain of recognizing illegal chess positions in the KRK end game [19]. The goal is to learn the concept of an illegal white-to-move position with only white king, white rook and black king on the board. The goal predicate is illegal (A, B, C, D, E, F) where the parameters correspond to the row and file coordinates of the pieces in the above order. Background knowledge consists of the predicates $X<Y, X=Y$ and adjacent $(X, Y)^{3}$. Recursion was not allowed for efficiency reasons.

Class noise in the training instances was generated according to the Classification Noise Process described in [2]. In this model a noise level of $\eta$ means that the sign of each example is reversed with a probability of $\eta .^{4}$ Noise was added incrementally, i.e. instances which had a reversed sign at a noise level $\eta_{1}$ also had a reversed sign at a noise level $\eta_{2}>\eta_{1}$. Similarly, training sets with $n$ examples were fully contained in training sets with $m>n$ examples.

In all experiments the induced rules were tested against sets of 5000 randomly chosen instances. It also proved useful to record the number of clauses in the induced concept and the average number of literals per clause to measure the complexity of the learned concept description.

\subsection{Finding a Good Cutoff Value}

The first series of experiments aimed at determining an appropriate value for this parameter for further experimentation. 10 training sets of 100 instances each were used at three different noise levels $(5 \%, 10 \%$ and $20 \%) .6$ different settings for the cutoff parameter $C$ were used. The results averaged over the 10 runs are reported in Fig. 1.

The following observations can be made from these graphs:

- A good setting for $C$ in this domain seems to be somewhere around 0.3 for all three noise levels. Coincidentially, the learned concepts are of about equal complexity at this point.

- The curve for the predictive accuracy is U-shaped, similar to some results from Decision Tree learning (see e.g. [4]).

- There is a transition from overfitting the noise to over-generalizing the rules. A low setting of $C$ has a tendency to fit the noise, because most of the literals

3 Because of a misunderstanding our definition of adjacent actually was adjacent_or_equal. However, changing this definition will not change the qualitative results reported in this paper.

4 Note that this differs from some of the results in the ILP literature, where a noise level of $\eta$ means that, with a probability of $\eta$, the sign of each example is randomly chosen. Thus a noise level of $\eta$ in our experiments is roughly equivalent to a noise level of $2 \eta$ in the results reported e.g. in $[11,10]$. 

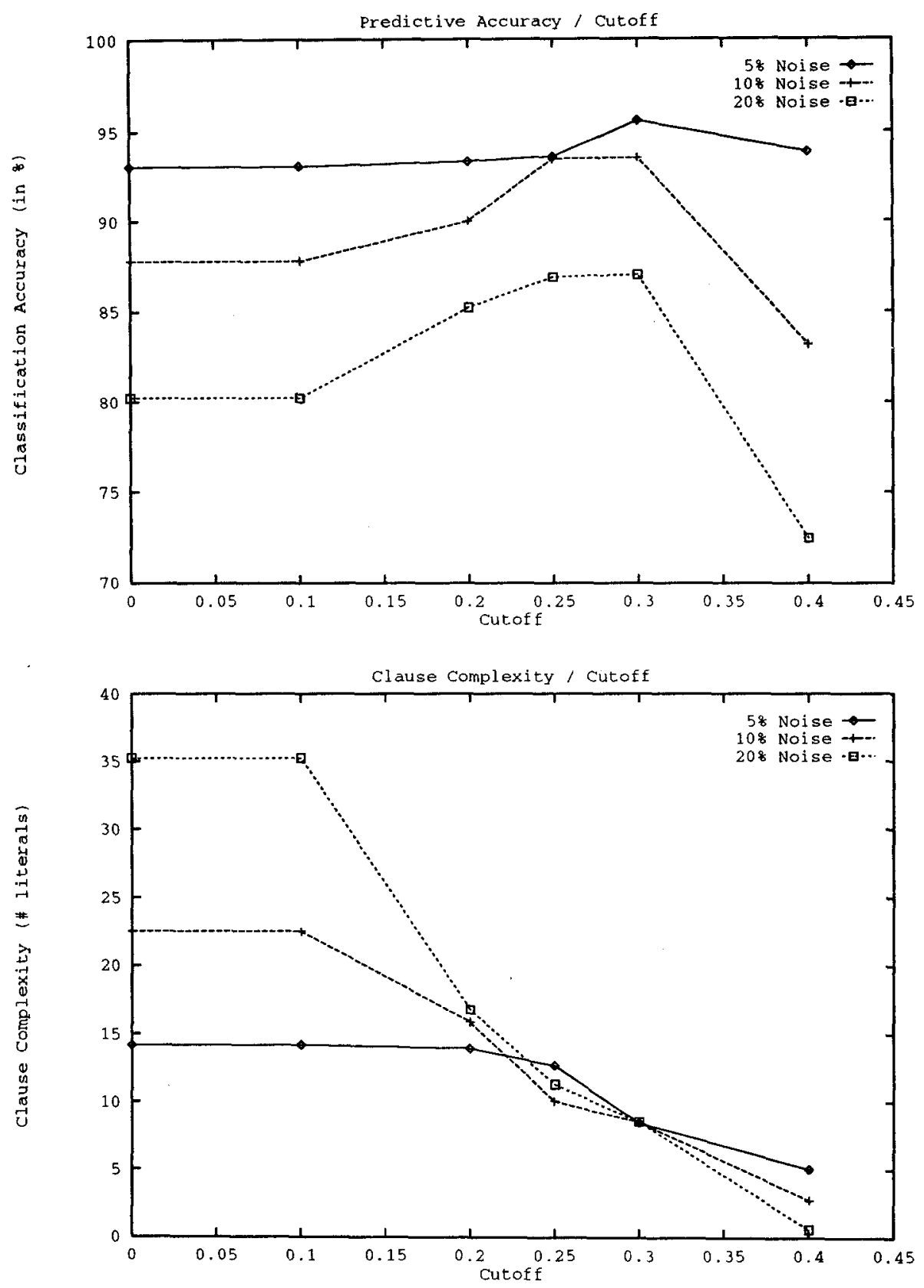

Fig. 1. Experiments with different settings for the Cutoff 
will have a correlation above the threshold. ${ }^{5}$ Conversely, a too optimistic setting of $C$ results in over-generalization as too few literals have a correlation above the threshold.

- The complexity of the learned concepts monotonically decreases with an increase of the cutoff parameter.

- The influence of a bad choice of the cutoff is more significant in data containing a larger amount of noise.

\subsection{Comparison with FolL}

We performed two experiments to compare FossiL's performance to the performance of FoIL. In the first series we compared the behavior of the two systems with 10 training sets of 100 instances each at different noise levels, which has been the standard procedure for evaluating many ILP systems $[20,11,10,19]$. In the second experiment we evaluated both programs at a constant noise level of $10 \%$, but with an increasing number of training instances.

According to the results of the previous experiments we set $C=0.3$ and never changed this setting.

Comparison at Different Noise Levels. In this experiment we compared Foll4 to Fossil at different noise levels. In order to have a fair comparison to FossiL where backtracking is not implemented, we used two versions of FoIL, regular Foll4 and a new version, FolL-NBT, where Foll4's extensive mechanisms of backing up and regrowing clauses [23] were not allowed. Surprisingly this version performed better than the original Foll 4 in noisy data as can be seen from the results of Table 1 .

An analysis of the result shows that FossiL performs best in most of the tests, but no significant difference between Foll-NBT and FossiL can be found. A comparison of the average number of induced clauses and of the average literals per clause shows evidence that FossiL over-generalized at the high noise levels. A lower value of the cutoff parameter may result in better performance in the case of $30 \%$ noise, although it is unlikely that a useful theory would be learned. An interesting detail is that FossiL did not learn anything at a noise level of $50 \%$, i.e. with totally random data. Thus the cutoff mechanism seems to be a primitive, but efficient means of distinguishing noise from useful information.

On the other hand, FoIL4 seems to perform worse than both FoIL-NBT and FossiL. The complexity of the concepts learned by FoIL 4 increases with the amount of noise in the data, which is clear evidence for over-fitting noise in the data. The next experiment was designed to confirm this hypothesis.

Comparison at Different Training Set Sizes. In this series of experiments we compared FoIL-NBT to Fossil at different training set sizes, each having

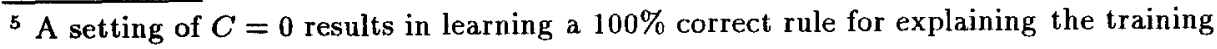
set. 
Table 1. A Comparison of Foll and FossiL on different levels of noise

\begin{tabular}{|c|c|c|c|c|c|c|c|c|c|}
\hline \multirow{2}{*}{\multicolumn{2}{|c|}{\begin{tabular}{|c|} 
Different \\
Noise Levels
\end{tabular}}} & \multicolumn{8}{|c|}{ Noise } \\
\hline & & $0 \%$ & $5 \%$ & $10 \%$ & $15 \%$ & $20 \%$ & $25 \%$ & $30 \%$ & $50 \%$ \\
\hline \multirow{3}{*}{ FoIL4 } & Accuracy & 98.32 & 95.26 & 92.12 & 90.26 & 85.21 & 79.83 & 71.53 & 53.00 \\
\hline & \# Clauses & 3.5 & 4.2 & 5.4 & 5.9 & 5.7 & 6.6 & 8.0 & 7.9 \\
\hline & Lits/Clause & 1.64 & 1.98 & 2.41 & 2.47 & 2.66 & 2.98 & 3.03 & 3.45 \\
\hline \multirow{3}{*}{ FoI-NBT } & Accuracy & 98.11 & 95.00 & $\overline{92.98}$ & 91.76 & 87.12 & 79.42 & 76.32 & 55.33 \\
\hline & \# Clauses & 3.5 & 4.1 & 4.2 & 4.2 & 4.5 & 5.4 & 5.0 & 5.2 \\
\hline & Lits/Clause & 1.64 & 1.98 & 2.34 & 2.48 & 2.67 & 2.80 & 2.79 & 3.08 \\
\hline \multirow{3}{*}{ FossiL $(0.3)$} & Accuracy & 98.54 & 95.57 & 93.52 & 92.83 & 87.00 & 81.63 & 70.59 & $(67.07)$ \\
\hline & \# Clauses & 3.7 & 4.3 & 3.8 & 4.2 & 3.2 & 2.7 & 0.7 & 0.0 \\
\hline & Lits/Clause & 1.62 & 2.02 & 2.24 & 2.29 & 2.67 & 2.69 & 0.85 & 0.0 \\
\hline
\end{tabular}

$10 \%$ noise. We decided to use FolL-NBT instead of Foll4, because it performed better in the previous series of tests. Besides, the version without backing up and regrowing clauses naturally runs faster, which proved to be important. However, we have done a few sample runs with Foll4 to confirm that its results would not be qualitatively different from those of FolL-NBT.

Again, we used 10 different training sets and averaged the results. The outcomes of these experiments are summarized in Fig. 2 (the Minimal Error curves will be explained in Sect. 5).

The most important finding is that ForL clearly fits the noise, while FosSIL avoids this and learns a slightly over-general, but much more useful theory instead. FolL's fitting the noise has several disadvantages:

Accuracy: The more examples there are in the noisy training set, the more specialized are the various clauses in the concept description, which decreases the predictive ability of each clause learned by Forl. ${ }^{6}$

Efficiency: Foll grows an increasing number of clauses with an increasing number of literals. Also, several of the literals chosen to fit the noise introduce new variables, which leads to an explosion of the size of the tuple set. In fact, the $\mathrm{C}$ implementation of FolL could complete none of the ten experiments with 2000 training examples within 500 minutes of CPU time, while the PROLOG implementation of FossiL only needed about 15 minutes of CPU time for each of the training sets, running on the same machine.

Understandability: It is a widely acknowledged principle that the more complex a concept definition is, the less understandable it will be, in particular when both definitions describe the same data set. While the descriptions induced by Foll for the large training sets were totally incomprehensible

${ }^{6}$ This Problem is known as the Small Disjuncts Problem [13] and has recently been addressed with an relational learning algorithm using probabilistic concept descriptions [1]. 

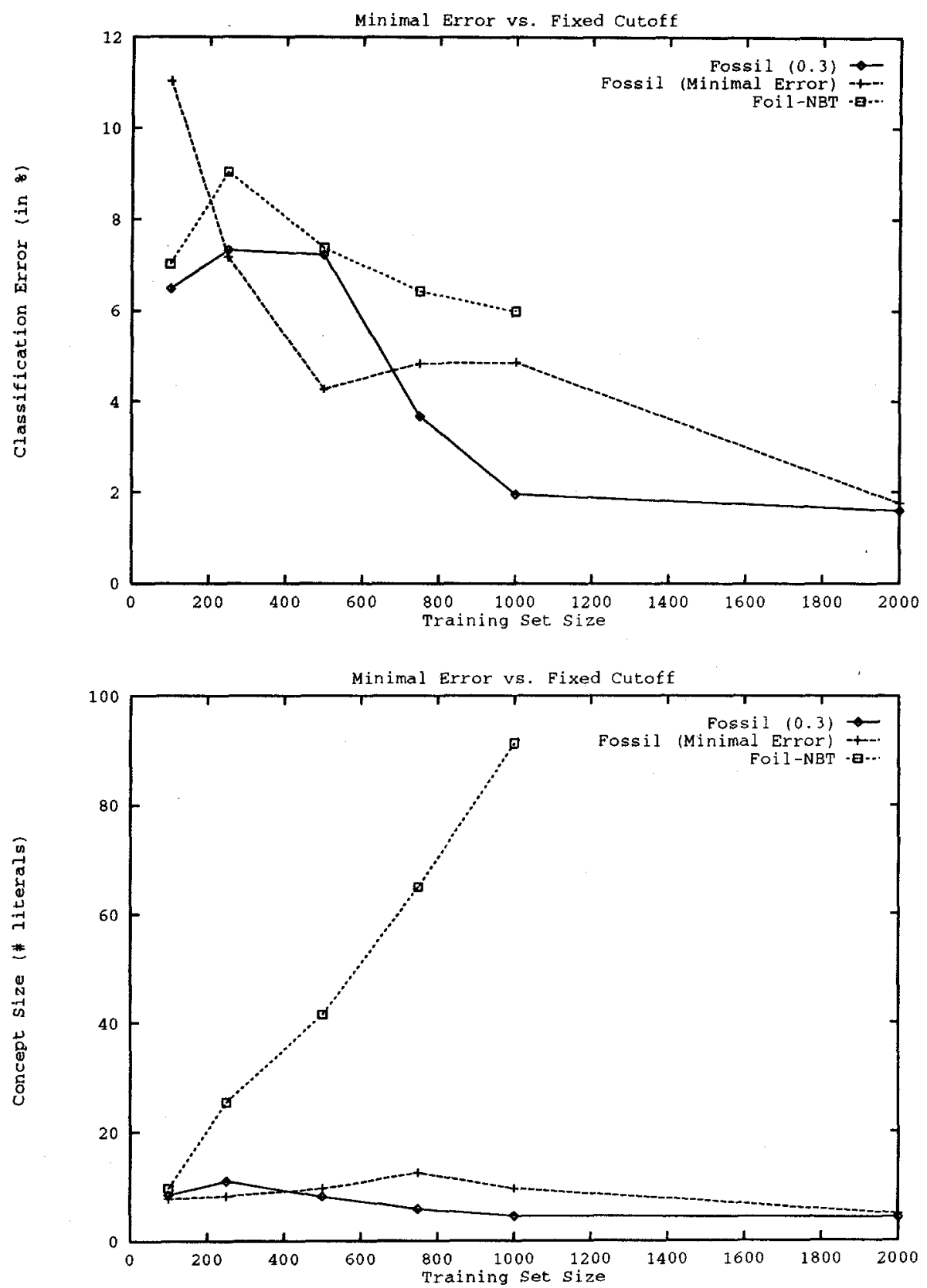

Fig. 2. A Comparison of Forl and FossIL with different training set sizes 


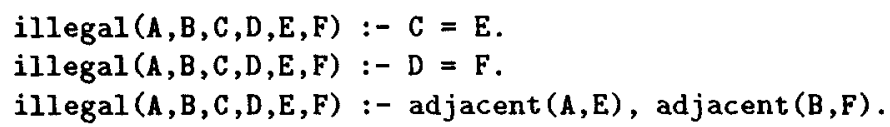

Fig. 3. An approximate theory that is $98.45 \%$ correct

to the author, FossiL converged towards the simple, approximate theory of Fig. 3. ${ }^{7}$ In fact, in 8 of 10 training sets with 2000 examples exactly this theory was learned, while in the other two the literal $\mathrm{A} \backslash==\mathrm{C}$ had been added to the first clause, which still gives a $97.98 \%$ correct theory [12].

What seems to be responsible for the drastic increase in the complexity of the learned clauses is that FoIL's stopping criterion [20] is dependent on the size of the training set. In the KRK domain it performs very well on sample sizes of 100 training examples. The more this number increases, the more bits are allowed for the theory to explain the data. However, more examples do not necessarily originate from a more complex theory. In fact, FoIL very often chooses the same literals as FossiL for the first clauses of its concept definition, but then continues to add literals and clauses, where FossiL stops.

FossiL uses a statistical stopping criterion based on the assumption that each literal in an explanation must have a significant correlation with the set of training examples. Statistical measures usually improve with the size of the training sets and so does the quality of the rules induced by FossiL. While both FolL and FossiL successively improve their predictive accuracy with increasing training set sizes, only FossiL converges towards a useful theory.

\subsection{Comparison with $m$ FolL}

$m$ FoIL [9] is an algorithm based on FoIL that has adapted several features from the CN2 learning algorithm, such as the use of the Laplace and m-estimate as a search heuristic and the use of significance testing as a stopping criterion [7]. These methods have proved very effective for noise handling. In addition $m$ FolL uses beam search (default beam width 5 ) and can make use of mode and type information to reduce the search space, features that are scheduled to be incorporated into FossiL in the near future. In our experiments $m$ FolL was used to its full capacity.

The values of the $m$ parameter were increased until a maximum performance was reached in the sets of 100 training examples. We then used the same values for testing with 1000 training examples. The results can be found in Table 2 .

7 This theory correctly classifies all but 4060 of the 262,144 possible domain examples (98.45\%). 2940 positions (1.12\%)with WK and WR on the same squares and 1120 positions $(0.43 \%)$ where the WK is between $W R$ and $B K$ on the same row or file are erroneously classified [12]. (Remember that we have defined adjacent to mean adjacent_or_equal). 
Table 2. Comparison with $m$ FoIL

\begin{tabular}{||c|ccccc|c||}
\hline Training & \multicolumn{5}{|c|}{$m$ FoIL } & FossIL \\
Set Size & $m=0.01$ & Laplace & $m=8$ & $m=16$ & $m=32$ & $C=0.3$ \\
\hline 100 & 89.77 & 89.84 & 93.03 & 93.06 & 91.46 & 93.52 \\
1000 & 91.54 & 92.51 & 95.70 & 97.10 & 98.48 & 98.05 \\
\hline
\end{tabular}

FossiL seems to be at least equal at an example size of 100 , unless a considerably better theory has been missed somewhere around $m=16$. However, $m$ FolL's strengths come to bear at an example size of 1000 . The results reported here are probably not yet the peak of its performance, as with $m=32 \mathrm{mFoIL}$ has learned some theories with a predictive accuracy of above $99 \%$ which FossiL has not achieved so far. ${ }^{8}$ Increasing the $m$ further might well improve the bad theories learned, while keeping the good ones.

However, one of the points to make here is that a good value of the $m$ parameter is not only dependent on the amount of noise (as can be seen from the results given in [9] and [10]), but also on the size of the example set. FossiL's cutoff parameter on the other hand seems to do reasonably well at different levels of noise and at different training set sizes.

In addition, Sect. 5 illustrates some preliminary results for finding good theories without having to specify a good value for the cutoff parameter.

\section{Generating a Series of Concept Descriptions}

As we have seen in Sect. 4.4, $\mathrm{mFOH}$ and Fossil have many similarities. However, a big disadvantage of $m$ Foll seems to be that it is not so easy to find the right $m$. The easiest approach is to try the standard settings used in the literature and choose the $m$ that results in the best theory according to an independent test set. However, with this approach one has no guarantee that one does not miss a better theory with a different $m$. The results given in [9] also indicate that the choice of a good $m$ depends on the amount of noise in the data, while our experiments in Sect. 4.4 also suggest a dependence on the size of the training set. FossiL, on the other hand, achieved reasonable results with one setting of the cutoff parameter on different noise levels as well as different training set sizes.

Another advantage of the cutoff stopping criterion is - besides its efficiency and stability - its close relation to the search heuristic. While Foll (encoding length restriction) and $m$ FoIL (significance test) have to do separate calculations to determine when to stop learning, FossiL needs to do a mere comparison between the heuristic value of the best candidate literal and the cutoff value. This allows the design of a very simple algorithm that can generate all theories

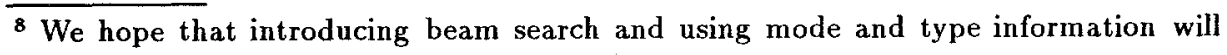
narrow the gap. 
that could be learned by FossiL with any setting of the Cutoff parameter (see Fig. 4).

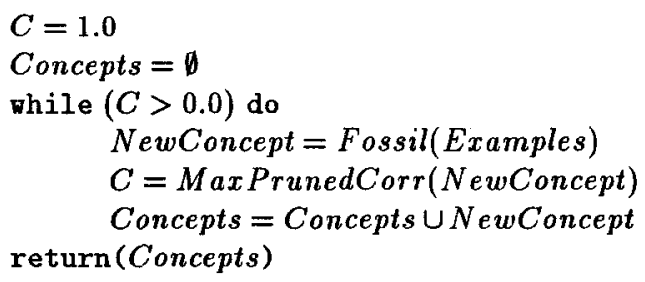

Fig.4. Algorithm to generate all Concept Definitions learnable by Fossn

The basic idea behind the algorithm given in Fig. 4 is the following: Assume that you are trying to learn a theory with a Cutoff of 1.0. Unless there is one literal in the background knowledge that perfectly discriminates between positive and negative examples, we will not find a literal with a correlation of 1.0 and thus learn an empty theory. During this run we can remember the literal with the maximum correlation. If we now set the new cutoff to exactly this maximum value, at least one literal (the one that produced this maximum correlation) will be added to the theory. ${ }^{9}$ At this new setting of the cutoff parameter we learn a new theory and again remember the maximum correlation of the literals that have been cut off. Obviously, for all values between the old cutoff and this maximum value, the same theory would have been learned and we can choose this value as the cutoff for the next run. It can also be expected that the new theory will be less general than the previous one. This process is repeated until we have the most specific theory (with MaxPrunedCorr $=0.0$ ). An illustration for this process can be found in Fig. 5, where a complete series of theories has been generated from 1000 noise-free examples of the KRK domain.

We have used this simple algorithm in the following way: The training sets were randomly split into two sets of equal size, one for training, one for testing. From the training set a series of theories was learned (all theories down to a cutoff of $0.15^{10}$ ) and from these the one with the best predictive accuracy on the test set was selected as the final theory. The results - labeled with Minimal Error - can be found in Fig. 2.

It can be seen that this naive and simple method performs better than ForL,

9 However, as our experience with FossiL shows, this is very often not the only change. Usually several more literals that have a correlation value higher than the new cutoff will be added. This is because literals that have a high correlation in the tuple sets covered by an incomplete clause or not covered by an incomplete concept do not necessarily have a high correlation with the tuples in the original training set.

10 This restriction was only made because of efficiency reasons. From our experience with previous tests we know that theories below 0.15 are usually very specialized and can be expected to give a high classification error. 

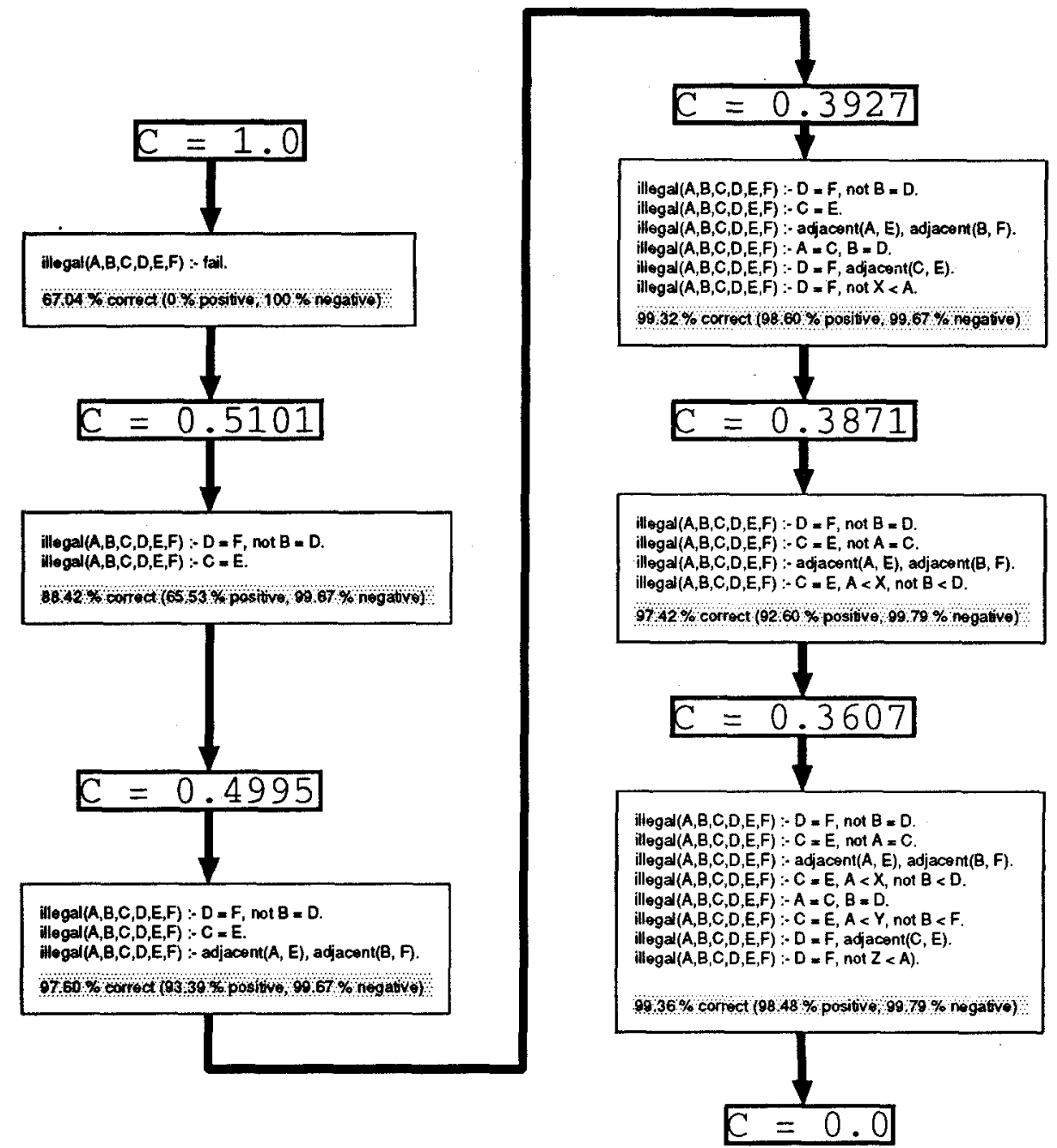

Fig. 5. Generating a series of theories in the noise-free KRK domain

although it practically only learns from half of the training examples. However, it is not as good as Fossil with a fixed cutoff. The fact that the minimal error method only uses half of the training examples for learning can also be seen from the graph, where FossiL's rather bad learning results at a size of 500 training examples reappear in the curve for the Minimal Error method at a training size of 1000 examples, although there is some improvement in the absolute numbers.

An analysis has also shown that the curves for classification accuracy are shaped similar to Fig. 1b, which suggests that some form of hill-climibing can be used to search this series of theories without having to generate all of them. (see Sect. 6). 


\section{Further Research: Top-Down Pruning}

While the naive approach of Sect. 5 might be too crude to be applied in this way, we do think that these preliminary results have some potential for refinement. In particular we see some relationship to pruning methods used e.g. in [5] or [25]. The major difference, however, is that we get a series of different concept descriptions in a general to specific order (top-down) as opposed to pruning methods the generate a most specific theory first and then successively generalize it (bottom-up).

We believe that the top-down approach has several advantages:

- With increasing example set sizes and increasing noise levels, generating a most specific starting theory for pruning becomes more and more expensive (as can be seen from the results of Foll in Sect. 4.3). Generating a simple general theory is much less expensive. In the experiments described in Sect. 5, typically less than 5 theories have to be generated to find the optimum and in particular the most specific and most expensive theories need not be learned.

- Efficiency can be further increased, as a clever implementation doesn't have to learn an entirely new theory. It can use the part of the last theory up to the point where the cutoff of the literal with the maximum correlation has occured.

- Pruning and learning are interleaved in this algorithm and can influence each other.

- In Decision Tree Learning several methods for selecting the best tree from a series of trees pruned to a different degree have been developed [17]. We hope that we can adapt some of these methods for relational learning and in particular make them "incremental", i.e. interleave them with the learning process in a way that generates as few unnecessary and expensive theories as possible.

- A weakness of all these algorithms is that they have to use part of the training set for pruning. Due to the robustness of the cutoff parameter we see a chance that a right value for the cutoff might be determined experimentally on parts of the learning set (e.g. with cross-validation) and that this information can be used to infer a good value for the parameter for learning from the entire set.

In the light of [24] a method like this may be viewed as automatically shifting the Overfitting Avoidance Bias, in some respect similar to CLINT, where a shift of Languate Bias is realized by learning in increasingly complex representation languages [8]. Both methods try to solve a problem in a simple way first CLINT by trying to use simpler representation languages first, and Top-Down Pruning by trying to find more general descriptions first - and subsequently switch to more complex solutions if necessary. 


\section{Conclusion}

The system described in this paper uses a new search heuristic based on statistical correlation along with a simple stopping criterion. We see the main advantages of this approach in its

Efficiency: There is no separate calculation of a heuristic function for negated literals and the amount of computing involved in calculating the stopping criterion is reduced to a mere comparison.

Robustness: A good value of the cutoff parameter seems to be independent of the amount of noise and the number of training examples.

Simplicity: In Sects. 5 and 6 we have outlined some promising approaches how the simplicity of the cutoff parameter and its close relation to the search heuristic might be used to interleave learning and pruning in a novel way.

However, $m$ Forl seems to do a little better in terms of classification error provided that one can find the optimal value of the $m$-parameter. Here we believe that implementing a simple beam search may help to narrow the gap.

\section{Acknowledgements}

This research is sponsored by the Austrian Fonds zur Förderung der Wissenschaftlichen Forschung (FWF) under grant number P8756-TEC. Financial support for the Austrian Research Institute for Artificial Intelligence is provided by the Austrian Federal Ministry of Science and Research. I would like to thank J. R. Quinlan and R. M. Cameron-Jones for making FoIl $4 \mathrm{ftp}$-able, Sašo Džeroski for providing $m$ Foll and a lot of patience, and Gerhard Widmer for a PROLOG implementation of the Foll algorithm to start with and for encouraging this research. Thanks are also due to two anonymous reviewers for their helpful comments.

\section{References}

1. Kamal M. Ali and Michael J. Pazzani. HYDRA: A noise-tolerant relational concept learning algorithm. In Proceedings of the Thirteenth Joint International Conference on Artificial Intelligence, pages 1064-1071, Chambèry, France, 1993.

2. D. Angluin and P. Laird. Learning from noisy examples. Machine Learning, 2(4):343-370, 1988.

3. Ivan Bratko and Igor Kononenko. Learning diagnostic rules from incomplete and noisy data. In B. Phelps, editor, Interactions in AI and Statistical Methods, pages 142-153, London, 1986.

4. L. Breiman, J. Friedman, R. Olshen, and C. Stone. Classification and Regression Trees. Wadsworth \& Brooks, Pacific Grove, CA, 1984.

5. Clifford A. Brunk and Michael J. Pazzani. An investigation of noise-tolerant relational concept learning algorithms. In Proceedings of the 8th International Workshop on Machine Learning, pages 389-393, Evanston, Illinois, 1991.

6. Wray Buntine and Tim Niblett. A further comparison of splitting rules for decisiontree induction. Machine Learning, 8:75-85, 1992. 
7. Peter Clark and Robin Boswell. Rule induction with CN2: Some recent improvements. In Proceedings of the 5th European Working Session of Learning, pages 151-163, Porto, Portugal, 1991.

8. Luc De Raedt and Maurice Bruynooghe. Indirect relevance and bias in inductive concept learning. Knowledge Acquisition, 2:365-390, 1990.

9. Sašo Džeroski and Ivan Bratko. Handling noise in Inductive Logic Programming. In Proceedings of the International Workshop on Inductive Logic Programming, Tokyo, Japan, 1992.

10. Sašo Džeroski and Ivan Bratko. Using the m-estimate in Inductive Logic Programming. In Logical Approaches to Machine Learning, Workshop Notes of the 10th European Conference on AI, Vienna, Austria, 1992.

11. Sašo Džeroski and Nada Lavrač. Learning relations from noisy examples: An empirical comparison of LINUS and FOIL. In Proceedings of the 8th International Workshop on Machine Learning, pages 399-402, Evanston, Illinois, 1991.

12. Johannes Fürnkranz. FossIL: A robust relational learner. Technical Report TR-9328, Austrian Research Institute for Artificial Intelligence, 1993. Extended version.

13. R. Holte, L. Acker, and B. Porter. Concept learning and the problem of small disjuncts. In Proceedings of the 11th International Joint Conference on Artificial Intelligence, Detroit, MI, 1989.

14. Nada Lavrač, Bojan Cestnik, and Sašo Džeroski. Search heuristics in empirical Inductive Logic Programming. In Logical Appraaches to Machine Learning, Workshop Notes of the 10th European Conference on AI, Vienna, Austria, 1992.

15. Nada Lavrač and Sašo Džeroski. Inductive Logic Programming: Techniques and Applications. Ellis Horwood, 1993.

16. Nada Lavrač, Sašo Džeroski, and Marko Grobelnik. Learning nonrecursive definitions of relations with LINUS. In Proceedings of the European Working Session on Learning, Porto, Portugal, 1991.

17. John Mingers. An empirical comparison of pruning methods for decision tree induction. Machine Learning, 4:227-243, 1989.

18. John Mingers. An empirical comparison of selection measures for decision-tree induction. Machine Learning, 3:319-342, 1989.

19. Stephen Muggleton, Michael Bain, Jean Hayes-Michie, and Donald Michie. An experimental comparison of human and machine learning formalisms. In Proceedings of the 6th International Workshop on Machine Learning, pages 113-118, 1989.

20. John Ross Quinlan. Learning logical definitions from relations. Machine Learning, 5:239-266, 1990.

21. John Ross Quinlan. Determinate literals in inductive logic programming. In Proceedings of the 8th International Workshop on Machine Learning, pages 442-446, 1991.

22. John Ross Quinlan. C4.5: Programs for Machine Learning. Morgan Kaufmann, San Mateo, CA, 1993.

23. John Ross Quinlan and R. M. Cameron-Jones. FOIL: A midterm report. In Proceedings of the European Conference on Machine Learning, pages 3-20, Vienna, Austria, 1993.

24. Cullen Schaffer. Overfitting avoidance as bias, Machine Learning, 10:153-178, 1993.

25. A. Srinivasan, S. H. Muggleton, and M. E. Bain. Distinguishing noise from exceptions in non-monotonic learning. In Proceedings of the International Workshop on Inductive Logic Programming, Tokyo, Japan, 1992. 